\title{
Retrograde balloon aortic valvuloplasty with the newly invented Inoue balloon for aortic stenosis accompanied by severe heart
}

\section{failure: a case report}

\author{
Keinchi Ishizu ${ }^{1}$, Tomohiro Kawaguchi ${ }^{2}$, Shinichi Shirai ${ }^{3}$, and Kenji Ando ${ }^{1}$ \\ ${ }^{1}$ Kokura Memorial Hospital \\ ${ }^{2}$ Kokura Kinen Byoin \\ ${ }^{3}$ Kokura Memorial Hsopital
}

January 5, 2021

\begin{abstract}
Balloon aortic valvuloplasty (BAV) is frequently utilized for selected high-risk patients who cannot be immediate candidates for transcatheter aortic valve replacement. We describe a case of high-risk retrograde BAV performed safely with a newly invented Inoue balloon with stable fixation and multistage inflation characteristics, without any rapid ventricular pacing requirements.
\end{abstract}

\section{Introduction}

Balloon aortic valvuloplasty (BAV) was introduced as an alternative to aortic valve replacement in 1986; it has played a limited role in the treatment of patients with severe aortic stenosis (AS) owing to complication risks, lack of durability, and its minor impact on long-term survival. ${ }^{1,2}$ However, in the era of transcatheter aortic valve replacement (TAVR), BAV is frequently utilized as a bridge procedure to TAVR for selected high-risk patients who cannot be immediate candidates for TAVR; thus, acute effectiveness and safety are required for BAV. ${ }^{3}$ Recently, the Inoue balloon for use in retrograde BAV (Toray, Chuo-ku, Japan) was newly invented. The hour-glass shaped balloon has multiple advantages, including stable fixation and multistage inflation characteristics, and therefore it does not impose any rapid ventricular pacing requirements to fix the balloon position during inflation, compared with cylindrical conventional balloons. We describe the BAV procedure which was safely performed using the newly invented Inoue balloon for retrograde approach in a high-risk patient.

\section{Case}

A 95-year-old woman with a history of hypertension was referred to our hospital with signs of progressive dyspnea. Upon arrival, she was tachypneic (respiratory rate: 27 breaths/min), hypotensive (blood pressure: 95/67 $\mathrm{mmHg}$ ), and tachycardic (heart rate: 110 beats/min). She had high-grade fever (body temperature: $38.5)$ and her oxygen saturation was $83 \%$ on room air. Physical examination revealed coarse crackling and wheezing in both lungs, a Levine IV/VI systolic murmur at the upper right sternal border, and bilateral lower extremity pitting edema. Laboratory test results were normal except for B-type natriuretic peptide levels of $5908.8 \mathrm{pg} / \mathrm{mL}$, creatinine levels of $1.97 \mathrm{mg} / \mathrm{dL}$, C-reactive protein levels of $4.2 \mathrm{mg} / \mathrm{dL}$, white blood cell count of $12,000 / \mu \mathrm{L}$, hemoglobin levels of $115 \mathrm{~g} / \mathrm{L}$, albumin levels of $23 \mathrm{~g} / \mathrm{L}$, and pyuria with nitrituria. A chest radiograph showed pulmonary congestion with a butterfly shadow and an increased cardiothoracic ratio (Figure 1A ). Transthoracic echocardiography revealed severe AS with severely decreased left ventricular ejection fraction (LVEF: 30.3\%) and stroke volume (SV index to body surface area: $22 \mathrm{ml} / \mathrm{m}^{2}$ ) (Video S1 ). The aortic valve area calculated with the continuity equation was $0.38 \mathrm{~cm}^{2}$ with a mean transaortic pressure 
gradient of $45.2 \mathrm{mmHg}$. Pulmonary hypertension was suggested by a systolic tricuspid regurgitation pressure gradient of $47.4 \mathrm{mmHg}$. Multidetector computed tomography demonstrated a severely calcified aortic valve with aortic annulus area of $360.3 \mathrm{~mm}^{2}$ with the maximum diameter of $25.4 \mathrm{~mm}$ and the minimum diameter of $17.8 \mathrm{~mm}$ (Figure 2 ).

The patient suffered from severe heart failure owing to low-flow-high-gradient AS with reduced LVEF and urinary tract infection. Noninvasive ventilatory support and intravenous furosemide administration were ineffective and urgent intervention for AS was required before the results of blood cultures were available. Our heart team dismissed the option of a surgical aortic valve replacement because of advanced age and highsurgical risk (Society of Thoracic Surgeons Predicted Risk of Mortality of 9.217\%). In addition, the patient was considered not to be an immediate candidate for TAVR because of the risks of subsequent endocarditis in the case of prosthesis implantation. Accordingly, we decided to perform a retrograde BAV as a bridge to TAVR.

Retrograde BAV was performed at day 3. To avoid rapid ventricular pacing considering the severely decreased LVEF, the newly invented Inoue balloon for retrograde BAV use was utilized (Figures 3A to 3C, Video S2 ). Systemic blood pressure decreased only during balloon inflation and recovered several seconds after balloon deflation (Figure 3D ). After six times inflations of the 20-mm Inoue balloon in the absence of rapid ventricular pacing, an adequate acute gain was achieved without any complications (Figures $3 E$ and $3 F$ ). Transthoracic echocardiography performed after the procedure showed improvement in LVEF to $41.1 \%$ and the aortic valve area of $0.60 \mathrm{~cm}^{2}$ with a mean transaortic pressure gradient of $36.9 \mathrm{mmHg}$. The patient's urine output immediately increased after BAV, and her heart failure was completely compensated at day 8 (Figure $1 B$ ). After the signs of infection improved, transfemoral-TAVR with the 23-mm SAPIEN 3 (Edwards Lifesciences, Irvine, California) was performed at day 23 (Video S3), and the patient was ambulatorily discharged after 4 days.

\section{Discussion}

Earlier reports on the feasibility of the newly invented Inoue balloon for retrograde BAV is scarce. Moriki et al. reported the hemodynamical stability during inflation of the Inoue balloon as pre-dilatation for TAVR ${ }^{4}$; however, there are no available reports regarding retrograde use of the Inoue balloon as a bridge BAV to TAVR. Generally, the Inoue balloon is extensively used for percutaneous transcatheter mitral commissurotomies or antegrade BAVs and has numerous advantages, including stable fixation, multistage inflation, and no requirements for rapid ventricular pacing, compared with conventional balloons. The antegrade BAV using the Inoue balloon reportedly resulted in a greater increase in the postprocedural valve area and a reduction in vascular complications and the risk for stroke, compared with the retrograde BAV using the conventional balloon. ${ }^{5}$ However, the antegrade approach itself is a more technically complicated and demanding procedure because it requires septal puncture and antegrade passage of a wire loop through the circulation. In addition, antegrade approach via femoral vein had lower accessibility to rescue TAVR than retrograde approach via femoral artery. Recently, the new Inoue balloon, which has a longer and thinner shaft, and a more elliptical tip, has invented and utilized for retrograde BAV in Japan.

In our case, we decided to urgently perform the retrograde BAV procedure with the Inoue balloon for three reasons. First, the patient was hemodynamically unstable owing to the severe AS and ongoing bacterial infection despite of the intensive medical therapy. Although there are no guidelines on TAVR for patients with bacterial infection, sepsis during the index TAVR hospitalization was reported to be associated with significantly higher rates of prosthetic valve endocarditis (PVE). ${ }^{6}$ BAV without the need for prosthetic implantation is a reasonable choice in our case in order to avoid subsequent PVE. Second, the patient's severe calcified aortic valve was risky for acute, significant aortic regurgitation post-BAV which could be resolved by rescue TAVR. Thus, we selected retrograde BAV with higher accessibility to transfemoral-TAVR than antegrade BAV. Third, the patient's LVEF was significantly reduced. Previous studies reported that a longer ventricular pacing duration was associated with morbidity and mortality, particularly in patients with low LVEF. ${ }^{7}{ }^{8}$ To avoid rapid ventricular pacing, the Inoue balloon rather than the conventional balloon was utilized in retrograde BAV for our case. 
We describe a feasible and safe retrograde BAV case using the newly invented Inoue balloon in a hemodynamically unstable patient having AS with reduced LVEF complicated with bacterial infection.

\section{Acknowledgement}

None

\section{Author Contribution:}

Kenichi Ishizu, MD (Data curation: Lead; Writing - original draft: Lead)

Tomohiro Kawaguchi, MD (Data curation)

Shinichi Shirai, MD (Writing - review \& editing: Lead)

Kenji Ando, MD (Supervision: Supporting)

\section{References}

1. Cribier A, Savin T, Saoudi N, Rocha P, Berland J, Letac B. Percutaneous transluminal valvuloplasty of acquired aortic stenosis in elderly patients: an alternative to valve replacement? Lancet 1986;1:63-67.

2. Ben-Dor I, Pichard AD, Satler LF, Goldstein SA, Syed AI, Gaglia MA Jr, Weissman G, Maluenda G, Gonzalez MA, Wakabayashi K, Collins SD, Torguson R, Okubagzi P, Xue Z, Kent KM, Lindsay J, Waksman R. Complications and outcome of balloon aortic valvuloplasty in high-risk or inoperable patients. J Am Coll Cardiol Intv 2010;3:1150-1156.

3. Moretti C, Chandran S, Vervueren PL, D'Ascenzo F, Barbanti M, Weerackody R, Boccuzzi G, Lee DH, de la Torre Hernandez J, Omedè P, Nijenhuis V, Igbineweka N, Lim P, ten Berg, Carriè D, HildickSmith D, Gulino S, Cannata S, Gargiulo G, Tamburino C, Conrotto F, Meynet I, Quadri G, Marangoni L, Taha S, Biondi-Zoccai G, Salizzoni S, Marra S, Gaita F. Outcomes of patients undergoing balloon aortic valvuloplasty in the TAVI era: A multicenter registry. J Invasive Cardiol 2015;27:547-553.

4. Moriki T, Tobaru T, Higuchi R, Shimizu J, Takanashi S, Takayama M. The brand-new Inoue balloon for retrograde approach: first experience in Japan. Cardiovasc Interv and Ther 2019;34,293-294.

5. Sakata Y, Syed Z, Salinger MH, Feldman T. Percutaneous balloon aortic valvuloplasty: antegrade transseptal vs. conventional retrograde transarterial approach. Catheter Cardiovasc Interv 2005;64:314321.

6. Kolte D, Goldsweig A, Kennedy KF, Abbott JD, Gordon PC, Sellke FW, Ehsan A, Sodha N, Sharaf BL, Aronow HD. Comparison of incidence, predictors, and outcomes of early infective endocarditis after transcatheter aortic valve implantation versus surgical aortic valve replacement in the United States. Am J Cardiol 2018;122:2112-2119.

7. Fefer P, Bogdan A, Grossman Y, Berkovitch A, Brodov Y, Kuperstein R, Segev A, Guetta V, Barbash IM. Impact of rapid ventricular pacing on outcome after transcatheter aortic valve replacement. J Am Heart Assoc 2018;7:e009038.

8. Husser O, Holzamer A, Philipp A, Nunez J, Bodi V, Müller T, Lubnow M, Luchner A, Lunz D, Riegger GA, Schmid C, Hengstenberg C, Hilker M. Emergency and prophylactic use of miniaturized venoarterial extracorporeal membrane oxygenation in transcatheter aortic valve implantation. Catheter Cardiovasc Interv 2013;82:E542-E551.

\section{Legends}

Figure 1. Chest radiographs and the brain natriuretic peptide (BNP) levels before and after retrograde balloon aortic valvuloplasty (BAV)

(A) Preprocedural chest radiograph at day 1 showing severe pulmonary congestion with a butterfly shadow.

(B) Postprocedural chest radiograph at day 8 showing resolution of pulmonary edema.

Figure 2 . Multidetector computed tomography (MDCT) images of aortic complex 
Three-dimensional reconstruction of MDCT showing a severely calcified aortic valve with left ventricular outflow tract calcification (A) and aortic running (B). Multiplanar reconstruction of MDCT showing an aortic annular area of $360.3 \mathrm{~mm}^{2}(\mathrm{C})$, sinus of Valsalva diameter of $28.5 \mathrm{~mm}$ at the left coronary cusp, 28.0 $\mathrm{mm}$ at the right coronary cusp, $30.5 \mathrm{~mm}$ at the noncoronary cusp (D), and sinotubular junction maximum and minimum diameter of $25.7 \mathrm{~mm}$ and $25.0 \mathrm{~mm}(\mathrm{E})$.

Figure 3. Retrograde balloon aortic valvuloplasty using the Inoue balloon

Fluoroscopic images showing an inflated proximal portion of Inoue balloon (A), stable fixation of the Inoue balloon with its hourglass shape (B), and a fully inflated Inoue balloon (C). (D) Polygraph showing immediate recovery of blood pressure after balloon deflation. (E) Pressure tracing before BAV showing a mean pressure gradient of $51.5 \mathrm{mmHg}$. (F) Pressure tracing after BAV showing a mean pressure gradient of $32.2 \mathrm{mmHg}$. $\mathrm{AO}=$ aorta, $\mathrm{LV}=$ left ventricle

\section{Video S1.}

Transthoracic echocardiography on admission showing severe AS with severely decreased left ventricular ejection fraction.

\section{Video S2.}

Fluoroscopy showing retrograde balloon aortic valvuloplasty using the Inoue balloon. A proximal portion of the balloon was inflated first and its hourglass shape brought stable fixation.

\section{Video S3.}

Fluoroscopy showing transcatheter aortic valve replacement with the 23-mm SAPIEN 3 valve via transfemoral approach.

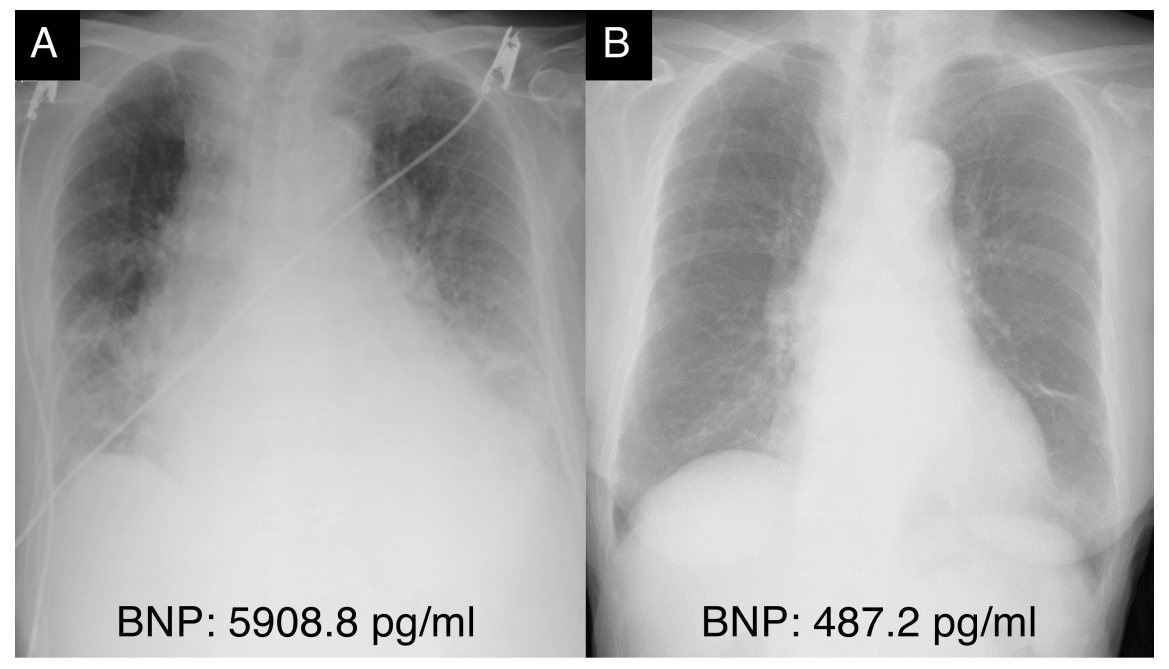



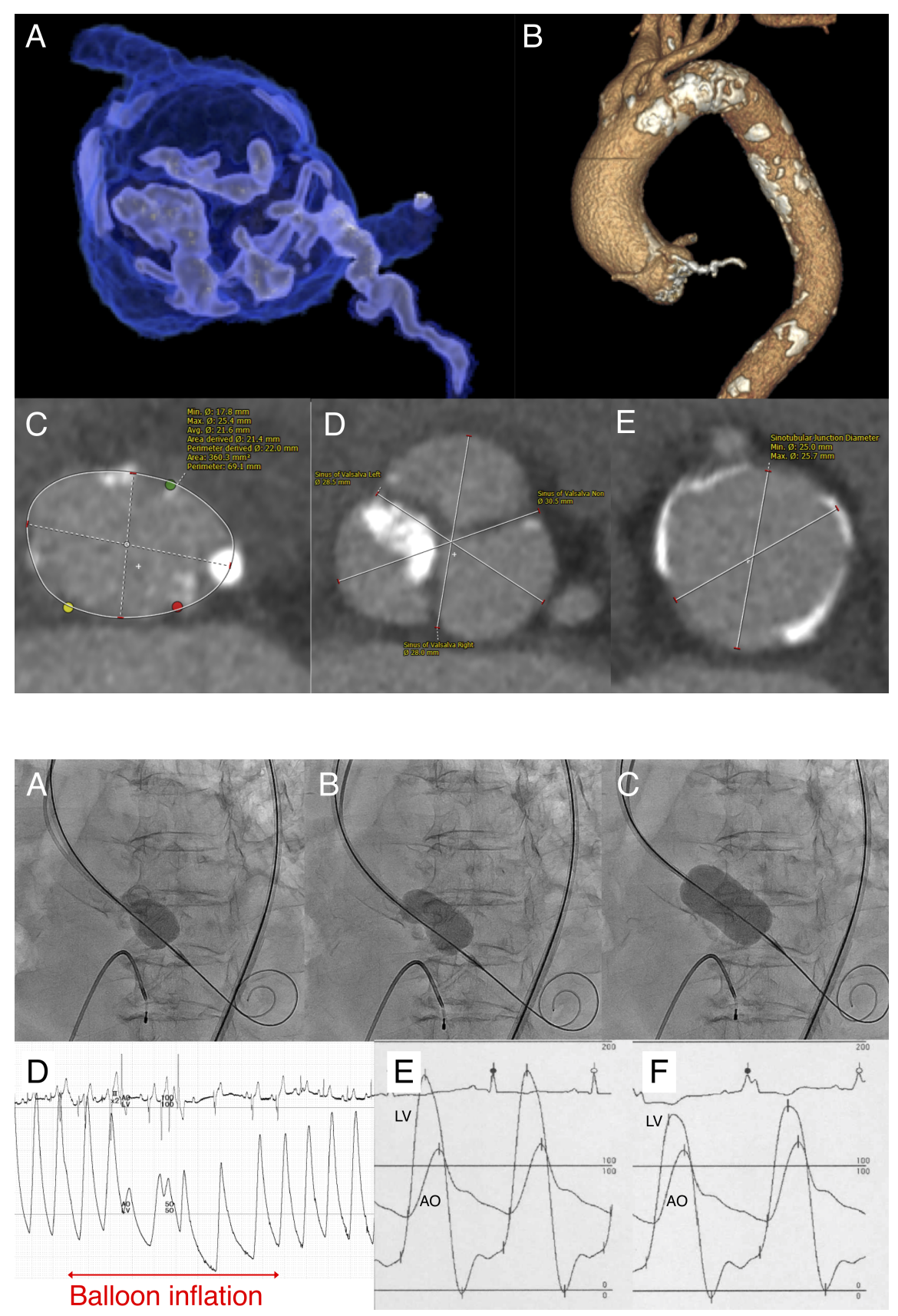